\section{Here I Am Again, Santa!}

\author{
Dear Santa,
}

I hope this season finds you in good health. Although I always begin with that, this year it has special meaning. Given that the world is in the midst of a pandemic, I imagine even the North Pole has its issues. It can't be easy for you to wear a mask over that big white beard, and you probably had to build a field factory so the elves could work safely at a distance. I hope you have a warm place for them to wash their hands; no sense risking frostbite! At least you all kept your jobs, because children everywhere need you more than ever this year.

This is a bit awkward to bring up, but I need to remind you that I have not received a single thing I've asked for the past few years. I know you sent a diamond bracelet last year since you couldn't figure out how to end unnecessary authorizations for medical care. That was a nice touch, and I am truly grateful. But honestly, can you get what I ask for, just once? I've been really good, I promise! I've followed all the usual precautions for COVID-19, and I didn't even get a black market haircut or a manicure. And I haven't attended any parties.

So here are my top 10 requests this year, in no particular order:

1. Give President-elect Joe Biden "medicine" to heal the country

2. Bring an effective COVID-19 vaccine

3. Fix "ObamaCare" so that it works the way it should and allows everyone decent healthcare coverage

4. Ensure that changes that have worked well, such as telehealth and reimbursements for time spent on a visit, continue after the pandemic

5. Bring an immunotherapy that works for all cancers

6. Give industry and the Centers for Medicare \& Medicaid Services a model for drug pricing that is rational so that medical care in America is affordable

7. Give everyone a mirror that allows us to see our own biases and begin to understand the need to change

8. Bring everyone a bag of patience, because nothing will change overnight

9. Deliver millions of new jobs so that the economy can recover

10. Bring me a diamond necklace to match the bracelet. Oops! Just kidding!

Well, that's my list. If you want to discuss any of this, I am always available for a Zoom meeting. We can include the elves if you like, but please ask them to stay on mute and just use the chat feature. They do have computers, don't they?

I'll end by wishing you and Mrs. Claus the very best of the season. And I'd like to thank you in advance for all the goodness I know you will pass around this holiday. The world is in a lot of hurt, and your spirit always makes everything better.

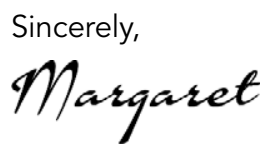
JNCCN@nccn.org or log into www.editorialmanager.com/JNCCN.

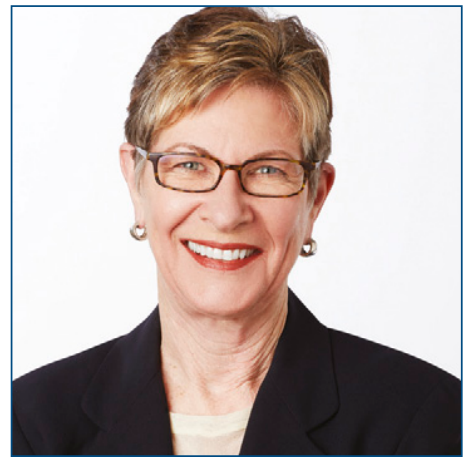

MARGARET TEMPERO, MD

Margaret Tempero, MD, is a Professor of Medicine and Director of the UCSF Pancreas Center and editor-in-chief of JNCCN. Her research career has focused on pancreatic ductal adenocarcinoma, especially in the area of investigational therapeutics. Dr. Tempero has served on the ASCO Board of Directors and as ASCO President. She currently serves on the ASCO Conquer Cancer Foundation Board. She codirected the AACR/ASCO Methods in Clinical Cancer Research and taught this course and similar courses in Europe and Australia. She was founding Chair of the $\mathrm{NCl}$ Clinical Oncology Study Section and served as a member and Chair of the $\mathrm{NCl}$ Board of Scientific Counselors Subcommittee A. She is a member of the Scientific Steering Committee and Chair of the Clinical and Translational Study Section for the Cancer Prevention \& Research Institute of Texas. She is or has been on the Scientific Advisory Boards of the Lustgarten Foundation, the Pancreatic Cancer Action Network, the V Foundation, The Alberta Canada Cancer Board, and the EORTC. She served as a member of the Oncology Drug Advisory Committee for the FDA. She has served as Deputy Director and Interim Director for the UNMC Eppley Cancer Center. She is Chief Emeritus of the Division of Medical Oncology at UCSF. She served as the founding Deputy Director and was later Director of Research Programs at the UCSF Helen Diller Family Comprehensive Cancer Center.

doi: $10.6004 /$ jnccn.2020.0059

The ideas and viewpoints expressed in this editorial are those of the author and do not necessarily represent any policy, position, or program of NCCN. 